\title{
CLOSED REDUCTION AND PERCUTANEOUS KIRSCHNER WIRE FIXATION COMBINED WITH PLASTER CAST VERSUS CONVENTIONAL PLASTER CAST IMMOBILIZATION IN THE TREATMENT OF COLLES' FRACTURE - A PROSPECTIVE RANDOMIZED COMPARATIVE STUDY
}

\author{
UZZAMAN KS ${ }^{1}$, AWAL KA ${ }^{2}$, ALAM MK $^{3}$
}

\begin{abstract}
:
Purpose- To evaluate functional \& anatomical results of the Colles' fracture treated by two methods- i) Closed reduction \& precutaneous kirschner wire (K-wire) fixation combined with plaster cast \& ii) Conventional plaster cast immobilization after closed reduction.

Methods- A randomized prospective comparative study was done from July 2003 to June 2005 on 52 patient with colles' fracture in NITOR, Dhaka. 12 patients lost from follow up. Twenty patient (20) in each group were finally available for evaluation. The group treated by closed reduction \& percutaneous $K$-wire fixation combined with plaster cast- designated as "Arm A" \& another group treated conventionally by plaster cast only designated by "Arm-B". Male Female ratio was 1:3, Age range was 35 to 70 years (Mean age 52.5 years) Distribution of limbs side involvement almost equal. Mode of injuries were domestic fall \& RTA (4:1) According to $A O$ classification fracture were in both groups- $A_{2}=13, A_{3}=10, C_{1}=6, C_{2}=8 \& C_{3}=3$ (Total40). Follow up period was 6-14 months (mean $=6$ month)

Results- Union time for most of the fractures was 6-8 weeks. At final follow up Satisfactory Anatomical end results of percutuneous fixation group (Arm-A) were $80 \%$ and in conventional plaster cast group $(A r m-B)$ were $35 \%$. $(P<0.01)$. Satisfactory functional end results in Arm- $A$ group were $70 \%$ and in Arm-B group were $30 \%(P<0.01)$ Complications seen much more in conventional group (Arm-B) than percutaneous $K$-wire fixation group (Arm-B). Sarmiento \& Latta's criteria was used to evaluate the progress.

Conclusion: The coventional plaster cast method for treatment of colles' fracture usually can't maintain radial length \& angulation in many instances and results significant anatomical difficultly and functional disability. On the other hand after close reduction additional fixation in the form of percutaneous crossed $K$-wire can maintain the reduction till bony union \& prevent late collapse at fracture site \& provides better result.
\end{abstract}

Key Words: Colles' faracture, closed reduction, Plaster cast \& Kirschner wire (K-wire) fixation.

J Dhaka Med Coll. 2008; 17(2) : 98-105.

\section{Introduction:}

The Colles' fracture is the most common skeletal injury in persons over 40 years of age and especially in women. This suggest an association with osteoporosis and it represents about $60 \%$ of fracture of radius in this age group $^{1}$. These fractures are easily reduced by traction \& manipulation. This conventional closed manipulation and plaster cast immobilization is the common modality of treatment of colles' fracture. However maintenance of reduction in a plaster cast alone is not always possible and invariably which results in malunion and deformity. Not only cosmetic result is disappointing but also disastrous results with collapse occurring

1. Assistant Professor, Orthopaedic Surgery, Shaheed Suhrawardy Medical College, Dhaka.

2. Professor of Orthopaedic Surgery \& Director, NITOR, Dhaka.

3. Assistant Professor, Orthopaedic Surgery, DMCH, Dhaka.

Correspondence: Dr. Kazi Shamimuzzaman 
within ten days in almost all fractures due to metaphyseal bone loss. ${ }^{1}$

Displaced, comminuted colles' fracture remain challenging problems of othorpaedic surgeons as they tend to heal with malunion. The conventional method of treatment can not maintain alignment as regards radial length and angulation in many instances and results in significant anatomic deformity and functional disability. Dorsal angulation can disturb radio-carpal function and reduces the range of motion. Decreased radial inclination or radial shortening may results in poor grip strength. Most authors agree that maintaining radial length- is one of the most crucial factor in regaining function of the wrist and the shortening of greater than 4 to $6 \mathrm{~mm}$ compromises function especially at the distal radio ulnar joint.

Colles' fractures have an inherent tendency for loss of reduction unless measures are taken to prevent redisplacement. This sorts of instability can be recognized by the presence of: Much comminution, Severe dorsal angulation $\left(20^{\circ}\right.$ or More), Radial shortening 10 $\mathrm{mm}$ or More, Extensive intra articular involvement $^{3}$

There are various methods that reduces the residual deformity by maintaining the reduction with additional fixation have been reported to improve the result ${ }^{4}$. These methods include the use of:-

1) Percutaneous kirschner wire fixation with plaster cast immobilization.

2) External fixator application

3) Internal fixation by plate $\&$ Screw

4) Bone grafting $\&$

5) Bone cementing

But there is much disagreement as to the best modality.

In most colles' fracture loss of reduction is inevitable unless additional fixation is applied. Primary percutaneous kirschner wire fixation should be considered if the original fracture is unstable. Even with excellent reduction and adequate casting there is often gradual shortening at the fracture site as healing occurs. Ideally this should be prevented by adding kirschner wire fixation to cast support or by external fixation or open reduction $\&$ internal fixation ${ }^{5}$. It is found that after closed reduction-percutaneous kirschner wire fixation secure initial reduction and maintain radial length till bony union and prevent subsequent late collapse $\&$ other complications significantly. For this reason this fixation method is now considered for better result in the treatment of Colles' fracture ${ }^{6,7}$.

\section{Patients and Method}

It was prospective randomized controlled trail study using the data obtained through ongoing research to compare the results of closed reduction and percutaneous Krischner wire fixation and closed reduction with immobilization by plaster cast alone - in the management of Colles' fracture. Place of study : NITOR, Dhaka, Duration of the study : July 2003 to June 2005. From 52 patients 12 patients lost from follow up. Twenty patient (20) in each group were finally available for evaluation. 40 patients were selected by purposive sampling method as per inclusion $\&$ exclusion criteria $\&$ each sample was radomized by lottery. The group treated by closed reduction \& percutaneous K-wire fixation combined with plaster cast- designated as "Arm A" \& another group treated conventionally by plaster cast only designated by "Arm-B". Male Female ratio was $1: 3$, Age range was 35 to 70 years (Mean age 52.5 years) Distribution of limbs side involvement almost equal. Mode of injuries were domestic fall \& RTA (4:1) According to $\mathrm{AO}$ classification fracture were$A_{2}=13, A_{3}=10, C_{1}=6, C_{2}=8 \& C_{3}=3$ (in both group total 40 patient). Patients with displaced colles' fracture attending at the emergency and outpatient department of NITOR within 07 days of injuries, were included in this study. Follow up period was 6-14 months (mean $=6$ month).

Ethical Issue: This randomized controlled trial was done by the guide line of research protocol approved by the ethical committee of NITOR. And informed consent was taken from the patient $\&$ their families explaining about the treatment procedure, expected result and possible complications.

Arm A : Patient with displaced Colles' fracture (AO/ASIF type A2, A3, C1, C2 and C3) managed 
by closed reduction and two crossed percutaneous Kirschner wire fixation with plaster cast support. The procedure was done under Regional Block anesthesia(Brachial Plexus).

\section{Bio-mechanics of Kirshner wire Fixation:}

Colles' fracture - fixation by percutaneous Kirshchner wire described by Clancey ${ }^{4}$ is the best approach to treat these fractures. The critical point with respect to the management of fractures by utilizing Clancey's technique is that the volar cortex of distal radius fracture must be available to act as a fulcrum. Here two percutaneous - Smooth K-wire (1.6-1.8mm Diameter) are used as blocking pin-and left in place 6-8 weeks - depending on the geometry of fracture and quality of bone. ${ }^{8}$

In case of Colles' fracture - restoration of radial length - is usually not difficult to obtain by closed manipulation but it is difficult to maintain because the intact ulnar shaft acts as a pivoting point for the contracting strong radial muscles - especially the brachioradialis. In addition to this there is also some loss of radial length due to actual crushing of cancellous bone. Even with excellent reduction and adequate casting there is often - gradual shortening of radius at the fracture site as healing occurs - called Late Collapse. Ideally this is prevented by percutaneous K-wire fixation to cast support. ${ }^{5}$ In Colles' fracture percutaneous pinning usually provide sufficient fixation to minimize radial collapse and shortening and allows immobilization of the wrist at a neutral position within the cast. ${ }^{9}$ Which permits full motion of the fingers.

Arm B : Patients with same type of Colles' fracture managed by conventional methodreduction by closed manipulation and maintained by plaster cast immobilization. The procedure was done under general anesthesia.

Plaster was removed at $6^{\text {th }}$ week. K-wires were removed at 6-8 weeks depending on the radiological and clinical evidence of union.
Rehabilitation and follow up

Adequate rehabilitation program was started to regain functions and to prevent complications. Patient was requested to report weekly for first 3 weeks, three weekly for up to $12^{\text {th }}$ weeks, then monthly upto full or near full functional recovery. Advice of physical therapy department was obtained from time to time. At the final follow up patients were graded as excellent, good, fair $\&$ poor using the criteria of sarmiento \& Latta. Excellent \& good results were taken as satisfactory, fair $\&$ poor results were taken as unsatisfactory.

\section{Evaluation of results}

Anatomic results : Anatomic result of each procedure was graded and compared by radiological criteria outlined by Lidstrom as modified by Sarmiento and Latta ${ }^{10}$

Functional results : - Functional result of each procedure was evaluated and compared by functional criteria out lined by Gartland and Werley as modified by Sarmiento. ${ }^{11}$

\section{Results}

At the end of follow up the results were evaluated both radio logically and functionally with the help of prefixed criteria.

\section{Anatomical results:}

Anatomical results were evaluated by radiologic means in anteroposterior and lateral views of the involved wrist joint and then comparing with that of the normal side. Mean follow up time was 09 months (6 months to 14 months). Table vii shows that $16(80 \%)$ of the patients of ArmA had excellent and good Anatomical end result while that was in Arm-B was 7 (35\%). Statistical analysis has been done and found that $\mathrm{c}^{2}=$ 8.58 and $p=0.03(<0.05)$. It is statistically significant. So, the Anatomical end result of Arm-A was significantly better than Arm-B.

\section{Functional results :}

Functional results were based on the pointing system described by Gartland and Werley as modified by sarmiento. The table viii shows that $14(70 \%)$ of the patients of Arm-A had Satisfactory result. On the contrary, only 6 
(30\%) of the patients of Arm-B had the Satisfactory result. The statistical analysis shows that $\mathrm{c} 2=6.40$ and $\mathrm{p}=0.01(<0.05)$. So, there is statistically significant Satisfactory results in Arm-A than that of Arm-B.

The important complication observed in Percutaneous K- wire fixation group was pin tract infection in one patient which was controlled by antibiotic therapy after culture and sensitivity test and regular dressing. The important complications observed in conventional group were wrist and finger stiffness, Persistent wrist pain, reduced grip strength, late collapse \& reflex sympathetic dystrophy which were also improved by physical therapy.

\section{Table-I}

Age Distribution of the patients

\begin{tabular}{lcccc}
\hline & \multicolumn{2}{c}{ Arm-A $(\mathrm{n}=20)$} & \multicolumn{2}{c}{ Arm - B (n=20) } \\
Age & Frequency & $\%$ & Frequency & $\%$ \\
\hline$<40$ & 3 & 15 & 1 & 5 \\
$41-50$ & 8 & 40 & 7 & 35 \\
$51-60$ & 6 & 30 & 6 & 30 \\
$61-70$ & 3 & 15 & 6 & 30 \\
\hline Total & 20 & 100 & 20 & 100 \\
\hline
\end{tabular}

Table-II

Sex Distribution of the patients

\begin{tabular}{lcccc}
\hline & \multicolumn{2}{c}{ Arm-A } & \multicolumn{2}{c}{ Arm - B } \\
Sex & Frequency & Percent & Frequency & Percent \\
\hline Male & 6 & 30 & 5 & 25 \\
Female & 14 & 70 & 15 & 75 \\
\hline Total & 20 & 100 & 20 & 100 \\
\hline
\end{tabular}

Table-III

Distribution of mechanism of injury

\begin{tabular}{|c|c|c|c|c|}
\hline \multirow[b]{2}{*}{ Cause } & \multicolumn{2}{|c|}{ Arm-A $(n=20)$} & \multicolumn{2}{|c|}{ Arm $-B(n=20)$} \\
\hline & Frequency & Percent & Frequency & Percent \\
\hline Domestic & Fall 15 & 75 & 16 & 80 \\
\hline RTA & 5 & 25 & 4 & 20 \\
\hline Total & 20 & 100 & 20 & 100 \\
\hline
\end{tabular}

Table- IV

Distribution of types of fracture

\begin{tabular}{lcccc}
\hline Fracture & \multicolumn{2}{c}{ Arm-A $(\mathrm{n}=20)$} & \multicolumn{2}{c}{ Arm-B $(\mathrm{n}=20)$} \\
type & Frequency & $\%$ & Frequency & $\%$ \\
\hline A2 & 8 & 40 & 5 & 25 \\
A3 & 5 & 25 & 5 & 25 \\
C1 & 2 & 10 & 4 & 20 \\
C2 & 3 & 15 & 5 & 25 \\
C3 & 2 & 10 & 1 & 5 \\
\hline Total & 20 & 100 & 20 & 100 \\
\hline
\end{tabular}

Table-V

Distribution of Anatomical and functional result according to Sarmiento and Latta's Criteria (Arm-A)

\begin{tabular}{lcccc}
\multicolumn{7}{c}{$(n=20)$} & Fair & Poor \\
\hline Stage & Excellent & Good & 9 & 11 \\
\hline Pre-Operative & 0 & 0 & 3 & 2 \\
Post-Operative & 4 & 11 & 2 & 2 \\
Final Anatomical Score & 4 & 12 & 4 & 2 \\
Final Functional Score & 3 & 11 &
\end{tabular}

Table-VI

Distribution of Anatomical and functional result according to Sarmiento and Latta's Criteria (Arm-B) $(n=20)$

\begin{tabular}{lcccc}
\hline Stage & Excellent & Good & Fair & Poor \\
\hline Pre-Operative & 0 & 1 & 6 & 13 \\
Post-Operative & 1 & 15 & 2 & 2 \\
Final Anatomical Score & 1 & 6 & 7 & 6 \\
Final Functional Score & 1 & 5 & 9 & 5 \\
\hline
\end{tabular}


Table-VII

Comparison of Anatomical end result between two arms

\begin{tabular}{lcccc}
\hline \multicolumn{2}{l}{ Anatomical } & \multicolumn{2}{c}{ Arm-A } & \multicolumn{2}{c}{ Arm-B } \\
result & Frequency & $\%$ & Frequency & $\%$ \\
\hline Excellent & 4 & 20 & 1 & 5 \\
Good & 12 & 60 & 6 & 30 \\
Fair & 2 & 10 & 7 & 35 \\
Poor & 2 & 10 & 6 & 30 \\
\hline Total & 20 & 100 & 20 & 100 \\
\hline
\end{tabular}

Table- VIII

Comparison of Functional end result between two arms

\begin{tabular}{lcccc}
\hline Functional & \multicolumn{2}{c}{ Arm-A } & \multicolumn{2}{c}{ Arm-B } \\
end Result & Frequency & $\%$ & Frequency & $\%$ \\
\hline Excellent & 3 & 15 & 1 & 5 \\
Good & 11 & 55 & 5 & 25 \\
Fair & 4 & 20 & 9 & 45 \\
Poor & 2 & 10 & 5 & 25 \\
\hline Total & 20 & 100 & 20 & 100 \\
\hline
\end{tabular}

Table-IX

Comparison of the overall evaluation of the patients of both Arms (Arm-A and Arm-B)

\begin{tabular}{lcccc}
\hline & \multicolumn{5}{c}{ Arm-A (n=20) Arm-B (n=20) } \\
Result & Number & $\%$ & Number & $\%$ \\
\hline $\begin{array}{l}\text { Satisfactory } \\
\text { (Excellent \& Good) }\end{array}$ & 16 & 80 & 7 & 35 \\
$\begin{array}{l}\text { Unsatisfactory } \\
\text { (Fair and Poor) }\end{array}$ & 4 & 20 & 13 & 65 \\
\hline
\end{tabular}

Table shows that $16(80 \%)$ of the patients of ArmA had excellent and good Anatomical end result while that was in Arm-B was 7 (35\%). Statistical analysis has been done and found that $\mathrm{c}^{2}=8.58$ and $p=0.03(<0.05)$. It is statistically significant. So, the Anatomical end result of Arm-A was significantly better than Arm-B.

Table shows that $14(70 \%)$ of the patients of Arm-A had good and excellent outcome in terms of Functional end result and that of in case of Arm-B was 6 (30\%).

The table shows that $16(80 \%)$ of the patients of Arm-A had the satisfactory result. On the other hand in case of the patients of Arm-B it was 7 (35\%). Staistical Analysis has been done and shows that $\mathrm{c}^{2}=8.29$ and $\mathrm{p}<0.01$. The result of overall evaluation in indicates that there is significantly better Satisfactory result among the patients of Arm-A than Arm-B.

Percutaneous K-wire fixation (Arm-A)

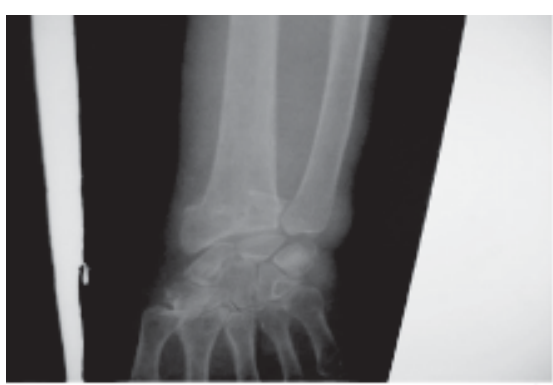

Pre-Operative

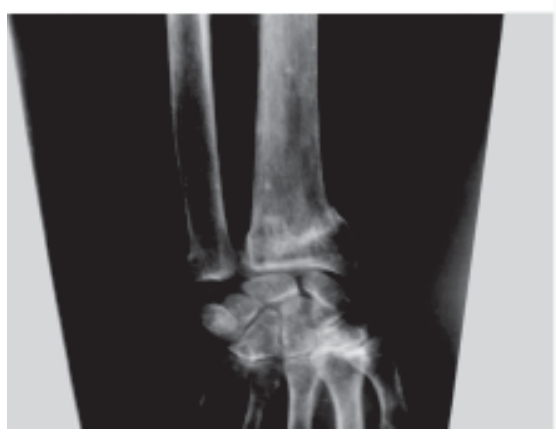

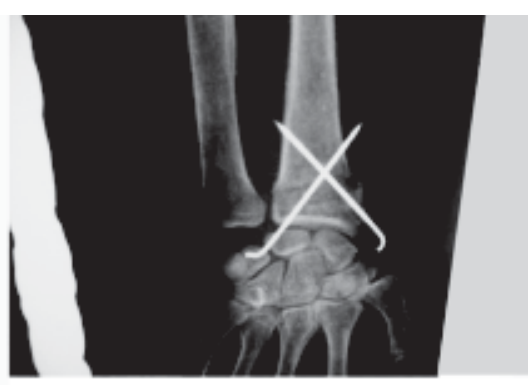

Post-Operative

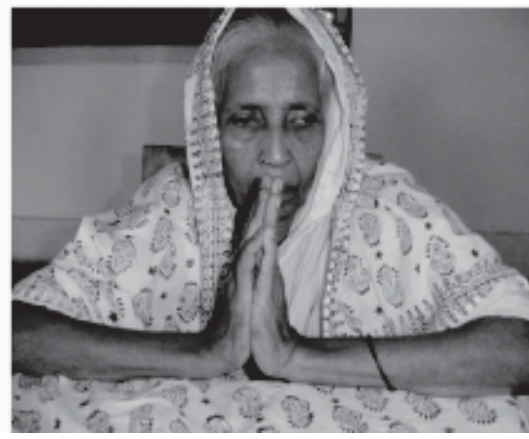

Satisfactory Alignment after 9 months Satisfactory function of the wrist after 9months 


\section{Conventional Plaster Cast (Arm-B)}
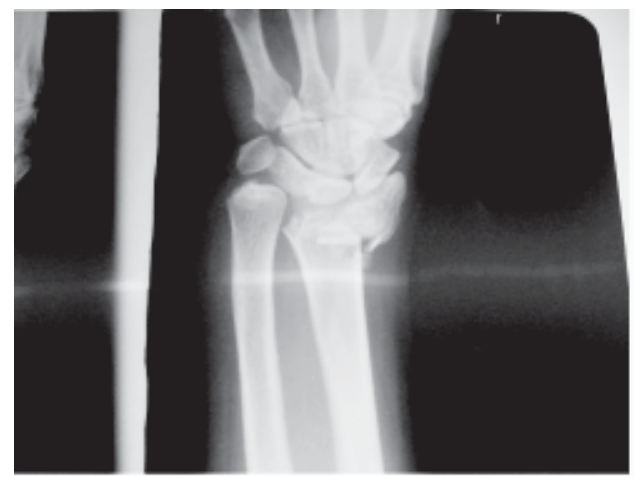

Initial X-ray

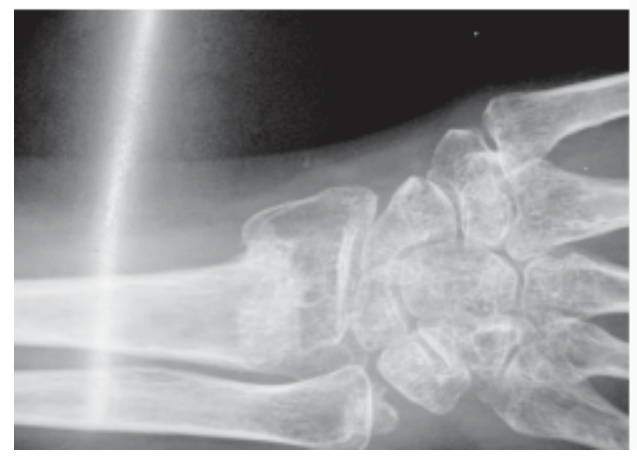

Unsatisfactory Alignment after 9 months

\section{Discussion}

The Colles' fracture is the most common skeletal injury in middle aged and elderly patient. But there is much conflict regarding the best modaility of treatment of this fracture. The conventional method of treatment usually can not maintain radial length and angulation in many instances and results in significant anatomic difficulty and functional disability. On the other hand after closed manipulation additional fixation in the form of percutaneous crossed $\mathrm{k}$-wire can maintain the reduction till bony union and provides better result. Though anatomical and functional results do not correlate completely, however outstandingly good anatomical restoration by fixation method invariably yields a fine functional result. Where as very poor anatomical results are associated with poor functional results. ${ }^{12}$ Abbaszadegan et al concluded that initial radial shortening in the best predictor of future instability, so the more importance has been given on the prevention of radial shortening there on the

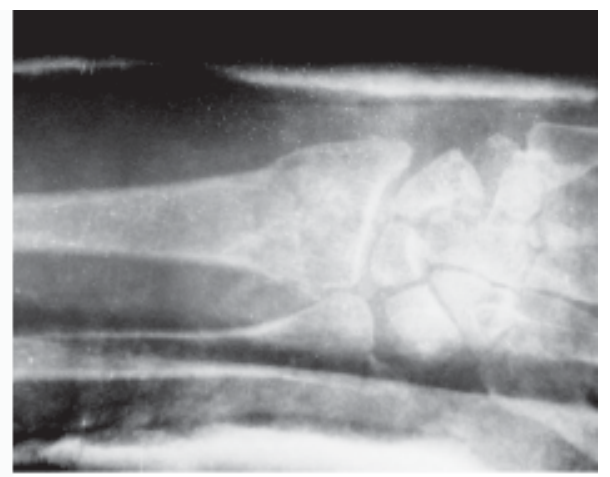

After close reduction \& plaster cast

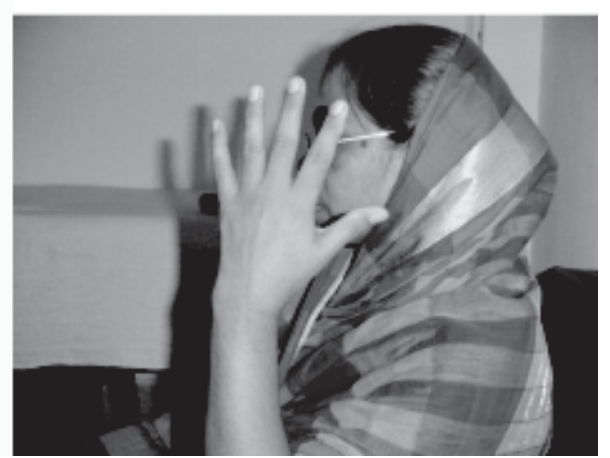

Unsatisfactory function of the wrist after 9months

reduction of dorsal angulation and radial angulation. ${ }^{13}$ It is not very difficult to achieve radial length by closed manipulation to the original value but in fairly difficult to maintain it before fracture healing is complete, especially if such fracture is protected by only cast immobilization.

Additional fixation offers the advantage of maintaining the reduction with the wrist in neutral position and allow full motion of fingers. Although the use of an external fixator is an effective means of achieving this result, it requires more specialized equipment and added expense and the bulk of the apparatus is cumbersome for elderly patient. Percutaneous $\mathrm{k}$-wire fixation is such a device that can serve this purpose very efficiently till healing of the fracture is complete. Many studies have substantiated this findings. ${ }^{2,3,5,14,15}$ The results in the present series also demonstrate that simple percutaneous fixation by two kirschner wire can successfully maintain reduction in most displaced Colles' fracture, with a very low 
incidence of complications and unsatisfactory results. In this series the highest number of patients belonged to $4^{\text {th }}$ and $5^{\text {th }}$ decade. Bracon and Kurtzke had shown in their study of two thousand cases that the average age of the patient was 48.02 years, ${ }^{2}$ very similar to my study. The commonest cause of Colles' fracture was fall on outstretched hand in most series which is also seen in my study. Post menopausal osteoporosis is the most important predisposing factor of Colles' fracture - which is also revealed in this study as the majority of patients $(>70 \%)$ were female in both groups. In my study it is found that the highest percentage of fracture type was AO/ASIF type A2 (40\%) and type C3 was the least in percentage in both the groups. But overall satisfactory results are more incase of percutaneous $\mathrm{k}$-wire fixation group (Arm-A) Which suggest that maintenance of reduction till complete healing with additional device is more important factor than the fracture pattern indetermining the final outcome. It was also supported by the study J. Clancey - in his series of Colles' fracture treatment. ${ }^{4}$ A number of complications were observed in my study in the both groups - but significantly less in case of percutaneous $\mathrm{k}$-wire fixation group. One patient of $\mathrm{k}$-wire fixation group had pin tract infection - which was managed by proper antibiotic after culture and sensitivity test. Two patient of this group also had proximal migration of $\mathrm{k}$-wires - which did not influences in maintaining the reduction achieved. It is seen in this study that during immobilization in plaster cast alone(Arm-B) the incidence of complications is much higher as that of ArmA. Among the patient of Arm-B there were loss of reduction in 12 patients which subsequently led to various disability like - persistent wrist pain, stiffness of wrist and finger, and reduced grip strength. It is also seen that late collapse in Arm-B group were in 10 patients (50\%) very similar to Gartland and Werley's series ${ }^{11}$ and in 2 patient of Arm-A after removal of procedure. This late collapse seen more in group $-B$ resulted in the more resident anatomic deformity functional disability at final outcome - in group-B which was also suggested by Bracon and Kurtzke. ${ }^{1,16}$ Yet the complications which are found after removal of procedures have been managed by adequate physiotherapy with the assistance from physical therapy department of NITOR. And within 3-6 month of physiotherapy there were substantial improvement regarding functional outcome. Anatomical results(Acc. To Sarmiento and Latta's score) of my study was satisfactory in $80 \%$ cases of percutaneous $\mathrm{k}$ wire fixation group whereas in conventional group it was $35 \%$. Functional results (Sarmiento \& Latta) in my study was satisfactory in $70 \%$ of percutaneous fixation group and 30\% in conventional group. All these anatomical and functional results correlate with the study of Max Scheck - where satisfactory results were in $75 \%$ case $^{4}$ and Gartland and Werley's series - where the satisfactory result was $70 \% .{ }^{11}$ Rodriguez Merchan E Carlos and Azzopardi T, Abela M showed, at their study of Colles' fracture treatment by both percutaneous pinning and plaster cast immobilization that the best anatomic and functional results were obtained by oercutaneous pinning 6,7 - which correlates with the results of my study. I agree with most of the author that correction and maintenance of anatomical land mark (radial length, radial angle, and dorsal angle) are the most important factors to regain hand and wrist function.

\section{Conclusion:}

Closed reduction and Percutaneous kirschner wire fixation combined with plaster cast immobilization is better method than the conventional plaster cast immobilization- in restoration of preinrury anatomical alignment and there by the functional outcome- in the management of colles' fracture.

\section{References:}

1. Robert W. Bacorm, John F Kurtke. Colles' fracture: A study of two thousands cases from New York state workmen's compensation Board; J Bone Joint Surg, Am. 1953; 35A,643-658.

2. Andrew H, Crenshaw, Jr. Fracture of shoulder girdle, arm and forearm; In Cambell's Orthopaedic; edited by Terrycanle, Memphis, Tennessee, 2003: 3058 - 3059.

3. Cooney WP, III, Linsched RL, Dobyns JH. External pin fixation of unstable Colles' fracture; J Bone Joint Surg Am, 1979; 61-A, 840-845. 
4. Clancey G J. Percutaneous kirschner wire fixation of Colles' fracture; J. Bone Joint Surg Am, 1984: 66-A, 1008-14.

5. Charls A Jr., Green DP. Fractures in adult, 4th edition, Vol-1: 769 -796.

6. Rodriguez- Merchan, Carlos. E. Plaster cast versus Percutaneous pin fixation for comminuted fractures of the distal radius in patients between 46 and 65 years of age, $J$ orthop Trauma; 1997:11 (3); April 212-217.

7. Azzopardi T, Ehrendorfer S, Coulton T, Abela M. A Prospective Randomized Study of Immobilization in a cast vs Supplimentary Percutaneous Pinning; J. Bone Joint Surg AM, June 2005.

8. Mattew, D Putnam. Fracture and Dislocation of the carpus including the distal radius. In fracture and Dislocation; Gustillo RB, 1993:558-581.

9. Ludvigsen TC, Johansen S. External Fixation vs Percutaneous Pinning for Unstable Colles' Fracture - An equal outcome; Acta Ortho Scandenivian; June 1997: 68(3), 255-258.

10. Lidstrom A: Fracture of the distal end of the radius. A clinical and statistical study of end results, Acta Orthop. Scand suppl; 1959;41(1).
11. Gartland JJ. Jr, Werley CW. Evaluation of healed Colles' fracture, J. Bone Joint Surg AM; 1951: 33-A; 895-907.

12. Dowling JJ, Sawyer B Jr. Comminuted Colles' Fractures : Evaluation of method of treatment $\mathrm{J}$ Bone Joint Surg [Am]; 1961: 34-A; 657-8.

13. Abbaszadegan H, Von-siversk, Jonsson U. Late displacement of Colles' fracture; int-ortho, 1988:12 (3): 197-9.

14. Joggenkong sholm and Clas Orlerud. Plaster cast versus external fixation for unstable intraarticular Colles' fracture, Clinical Orthopaedics and related research, Aug 1998 : No 241; 57-61.

15. Flin KKlin - T, Nikkole - Sil hota A, Raatikainen $\mathrm{T}$ : Role of metaphysical cancellous bone defect in secondary displacement of Colles'; Arch-orthopTrauma-Surgery; 1999: 119(5-9); 319-23.

16. Howard PPW, Stewart HD, Hind RE, Burker FD: External fixation or plaster of severely displaced comminuted Colles' fractures. A prospective study of anatomical and functional results. J.Bone Joint Surg [Br]; Jan 1989:71(1) 68-73. 